\title{
Dittrichia viscosa (L.) Greuter (Asteraceae): Nuevo registro para la flora introducida de Chile
}

\author{
Dittrichia viscosa (L.) Greuter (Asteraceae): New record for introduced flora of Chile
}

\author{
PATRICIO Novoa
}

Investigador asociado Jardín Botánico Nacional, Casilla 488, Viña del Mar, Chile pnovoa7@gmail.com

\begin{abstract}
Dittrichia viscosa is recorded for the first time in Chile as an introduced species. A brief description with photographs and, agrological antecedents are presented.
\end{abstract}

La familia Asteraceae (= Compositae) registraba en Chile 90 especies alóctonas asilvestradas (Marticorena, 1990). En 2011, Ugarte et al. informan la presencia de 118 especies de Asteráceas alóctonas asilvestradas pertenecientes a 68 géneros sobre la base de la revisión de los herbarios CONC y SGO y publicaciones de Marticorena \& Quezada (1985) y Matthei (1995). Asimismo Fuentes et al. (2013) registran la presencia de 106 especies de Asteráceas alóctonas. Es probable que la diferencia de doce especies entre ambos autores se deba a aspectos metodológicos y de fuentes de información por cuanto Ugarte et al. (2011) consultaron la base de datos de CONC, revisaron el herbario SGO y la investigación incluyó especies exóticas naturalizadas así como no autosustituidas a causa de introducciones humanas repetidas, por su parte Fuentes et al. (2013) usaron la base de datos del herbario CONC y publicaciones complementarias. A las especies registradas por Ugarte et al. (2011) y Fuentes et al. (2013) se suman Artemisia verlotiorum Lamotte, (Macaya \& Barriga 1995), Tragopogon dubius Scopoli (Macaya 2010) y los nuevos registros de Volutaria tubuliflora (Murb.) Sennem (Teillier et al. 2014) y Carduus tenuiflorus Curtis (Cordero et al. 2016).

En marzo de 2013 se encontraron 10 individuos florecidos de Dittrichia viscosa (L.) Greuter creciendo en un bandejón de rotonda del proyecto inmobiliario de Curauma (Placilla, Región de Valparaíso) frente a la zona de supermercados $33^{\circ} 07^{\prime} 49,42^{\prime \prime} \mathrm{S} ; 7^{\circ} 33^{\prime} 46,66^{\prime \prime} \mathrm{O}$, posteriormente en marzo de 2014 se encontró otra población de aproximadamente 1000 individuos florecidos en ambos bordes de la ruta 68 frente a la ciudad empresarial de Curauma $33^{\circ} 08^{\prime} 3,60$ ”s; 71³3’44,11'OO (Fig. 1A, 1B).

Estos registros constituyen la primera evidencia de su presencia en Chile pues no figura en los trabajos consultados en este artículo ni en la base de datos de la Flora del Cono Sur (Zuloaga et al. 2017).

El área de distribución nativa incluye las costas del sur de Europa (Bulgaria, España, Francia, Grecia, Italia), Turquía, Oriente Medio (Israel, Jordania y Siria) y el norte de África (Argelia, Egipto, Libia) (Brullo \& De Marco 2000).

Parolin et al. (2014) indican que Dittrichia viscosa tiene el potencial de ser una maleza agresiva debido a su eficiente producción y dispersión de semillas y a su buena adaptación a suelos perturbados y ecosistemas secos (Groves 1991). Es maleza introducida en las Islas Azores, Bélgica y Gran Bretaña (DAISIE 2012). Actualmente es común en otras regiones de Europa y Norteamérica. En Australia Occidental se considera una maleza ambiental grave y su importación está prohibida (Sinden et al. 2004, DPIPWE 2011). D. viscosa tiene una alta capacidad de crecimiento en suelos con altas concentraciones de níquel, magnesio o arsénico (Conesa et al. 2011, DPIPWE 2011) y fue propuesta como planta bioindicadora de estos elementos en el suelo (Ater et al. 2000, Parolin et al. 2014). Baker (1987) llama a D. viscosa un pseudometalófito porque puede crecer en suelos contaminados con metales pesados. Además, tiene un alto potencial como bioacumulador (Parolin et al. 2013).

D. viscosa actúa como planta hospedera en sistemas de cría y liberación de enemigos naturales de plagas agrícolas. Por ejemplo, puede ser hospedera útil de parásitos de áfidos en zonas agrícolas de Grecia (Kavallieratos et al. 2002), de ácaros fitoseideos (ácaros controladores de ácaros) en cultivos de vid en Francia (Tixier et al. 2000), de depredadores como Eupelmus urozonus que controlan la mosca Myopites stylata formadora de vesícula de la oliva en Andalucia, España (Franco-Micán et al. 2010, ITAB 2005,) E. urozonus es también uno de los principales parásitos de la principal plaga 

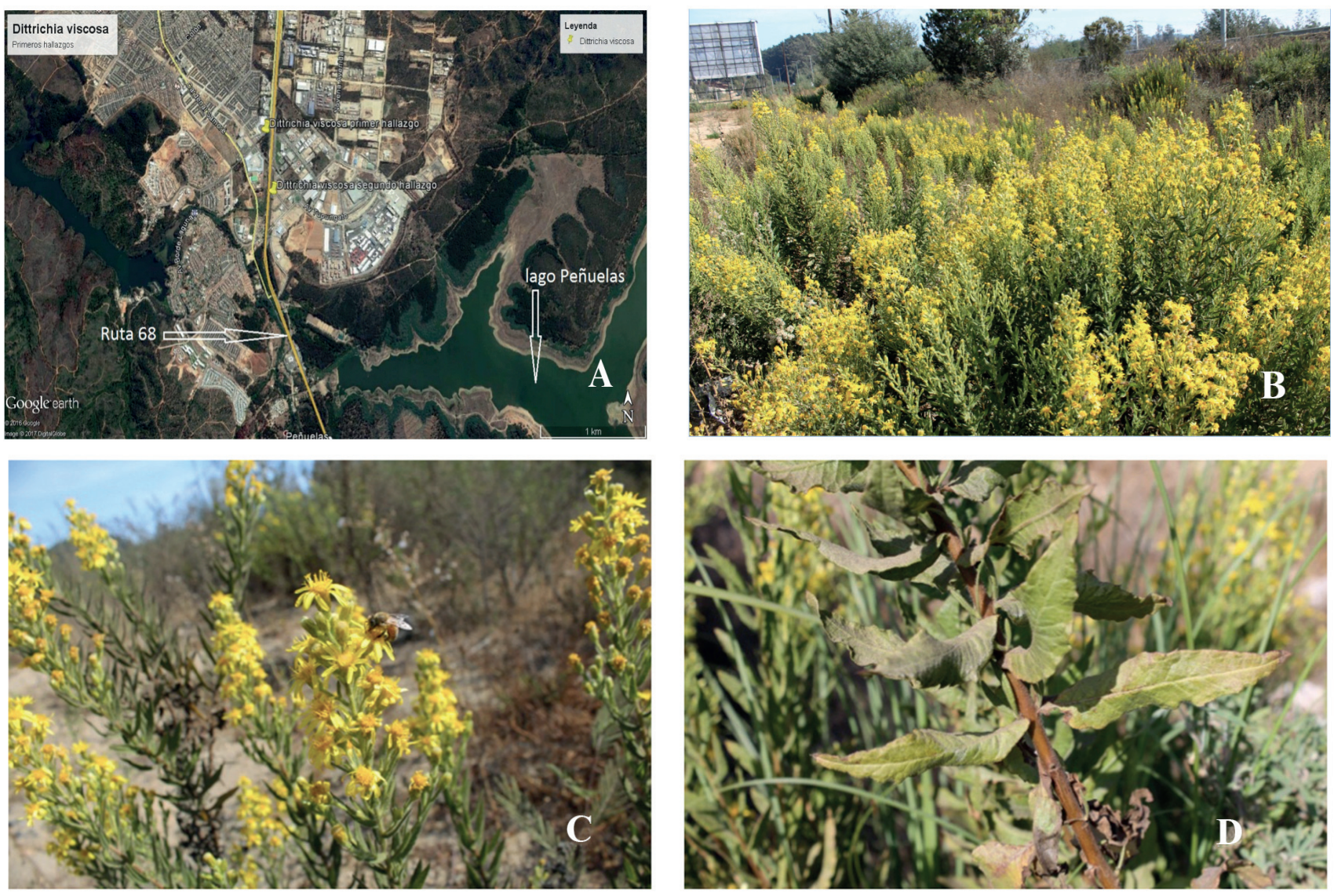

Figura 1. Dittrichia viscosa (L.) Greuter. A: inflorescencia. B: detalle hojas basales. C: población donde se puede observar numerosos individuos. D: mapa hallazgos de la especie. / Dittrichia viscosa (L.) Greuter. A: inflorescence. B: detail basal leaves. C: population where many individuals can be observed. D: map of species findings.

de la cosecha del olivo, la mosca de la aceituna Bactrocera oleae en Tanagra, Grecia (Broumas et al. 2002), Ditricchia viscosa es también hospedero de Macrolophus caliginosus (Miridae) depredador de plagas del tomate. Bajo ciertas condiciones puede actuar sobreinfectando plagas en cultivos (Parolin et al. 2013).

\section{DESCRIPCIÓN DE LA ESPECIE}

\section{Dittrichia viscosa (L.) Greuter}

Exsicc. Genav. 4: 71.1973

Arbusto hemicriptófito de hasta $150 \mathrm{~cm}$ de alto, con tallos erectos o ascendentes, muy ramificados en la base. Hojas oblongo-lanceoladas, sésiles, láminas de (10-) 20-80 (-100) x (3-) 6-27 mm, margen subentero a marcadamente aserrado; las caulinares semiamplexicaules. Involucro de (5,5-) 6,5-10 $\mathrm{mm}$ de largo. Flores femeninas con lígulas con dientes apenas marcados (hemiliguladas) de (7-) 8-11,6 $\mathrm{mm}$ de largo, amarillas, a veces con el dorso ligeramente teñido de púrpura, sobrepasando al involucro. Flósculos hermafroditas de 5-7 mm de largo, de corola amarilla, rara vez teñidos de púrpura. Cipselas de las flores flosculosas de 2-2,6 (-3) mm de largo, con vilano de 5-6,2 (-6,5) $\mathrm{mm}$ de largo. $2 \mathrm{n}=18,24$ (Fig. 1C, 1D). Florece desde mediados de enero a abril, y fructifica de abril a mayo. En España florece $\mathrm{y}$ fructifica de agosto a noviembre (enero).

Material estudiado: CHILE, Región de Valparaíso, Provincia de Valparaíso, Placilla ciudad empresarial Curauma, 338 m. 23-III-2014. Patricio Novoa 1268 (CONC 183539).

\section{REFERENCIAS}

Ater, M., Lefèbvre, C. Gruber, W., Meerts, P. 2000. A phytogeochemical survey of the flora of ultramafic and adjacent normal soils in North Morocco. Plant and Soil 218: 127-135. 
BAKer, A.J. 1987. Metal tolerance. New Phytologist 106: 93-111.

Brullo, S., De Marco, G. 2000. Taxonomical revision of the genus Dittrichia (Asteraceae). Portugaliae Acta Biologica 19(1): 341-354.

Broumas, T., Haniotakis, G., Liaropoulos, C., Tomazou, T., Ragoussis, N. 2002. The efficacy of an improved form of the masstrapping method, for the control of the olive fruit fly, Bactrocera oleae (Gmelin), Dipt., Tephritidae. Pilot scale feasibility studies. Journal of Applied Entomology 126: 217-223.

Conesa, H.M., Cervantes, A., Alvarez, J., Gonzalez, M. 2011. Influence of soil properties on trace element availability and plant accumulation in a Mediterranean salt marsh polluted by mining wastes: Implications for phytomanagement RID A-3481-2009. Science of the Total Environment 409: 4470-4479.

Cordero, S., Gutiérrez, D., Moreira-Muñoz, A. 2016. Carduus tenuiflorus Curtis (Asteraceae), nuevo registro para la flora alóctona de Chile. Gayana Botánica 73: 453-456.

DAISIE 2012. Citing Electronic Resources. Delivering Alien Invasive Species Inventories for Europe, URL: http://www. europe-aliens.org/speciesFactsheet.do?speciesId=22359\#. Accedido: Abril 2017.

DPIPWE (Department of Primary Industries, Parks, Water and Environment). 2011. False Yellow Head Weed Management Plan. Statutory Weed Management Plan as at June 2011. Tasmania / Australia. URL: http://www.dpiw. tas.gov.au/inter.nsf/Attachments/LBUN-8EZ5HA?open. Accedido: Abril 2017.

Franco-Micán, S., Castro, J., CAmpos, M. 2010. Preliminary study of the parasitic complex associated with Dittrichia viscosa in Andalusia (Spain). IOBC/WPRS Bulletin 53: 139-143.

FreIre, S. 2015. Flora vascular de la República Argentina 7(2): Dicotyledoneae-Asteraceae (Cichorieae, HelenieaeMutisieae). (Zuloaga F., Belgrano M., \& Anton A., Eds.). Instituto de Botánica Darwinion.

Fuentes, N., Pauchard, A., Sánchez, P., Esquivel, J., Marticorena, A. 2013. A new comprehensive database of alien plant species in Chile based on herbarium records. Biological Invasions 15: 847-858.

GRoves RH 1991. The biogeography of mediterranean plant invasions. In: Groves, R.H., Di Castri, F. (eds.) Biogeography of mediterranean invasions: 427-438. Cambridge University Press, Cambridge, United Kingdom.

ITAB. 2005. Produire des fruits en agriculture biologique. Alter Agri 72: 1-32.
Kavallieratos, N.G., Stathas, G.J., Athanassiou, C.G., Papadoulis, G.T. 2002. Dittrichia viscosa and Rubus ulmifolius as reservoirs of aphid parasitoids (Hymenoptera: Braconidae: Aphidiinae) and the role of certain coccinellid species. Phytoparasitica 30: 231-242.

Macaya, J. 2010. Tragopogon dubius Scopoli (Asteraceae), un nuevo registro para la flora adventicia de Chile. Chloris Chilensis, Año 13(2). URL: http://www.chlorischile. $\mathrm{cl} /$ tragopogon-Macaya/macaya-TRAGOPOGON\%20 dubius.htm

MACAYA, J., BARRIGA, J. 1995. Nueva especie de maleza encontrada en Chile, Artemisia verlotiorum Lamotte (Asteraceae). Investigación Agrícola 15(1-2). Enero - diciembre 1995.

Matthei, O. 1995. Manual de las malezas que crecen en Chile. Alfabeta Impresores, Santiago. $545 \mathrm{pp}$.

Marticorena, C. 1990. Contribución a la estadística de la flora vascular de Chile. Gayana Botánica 47: 85-113.

Marticorena C., M. Quezada. 1985. Catálogo de la flora vascular de Chile. Gayana Botánica 42: 1-158.

Parolin, P., Bresch. C., Ottenwalder, L., Ion-Scotta, M., Brun R., Fatnassi, H., Poncet, C. 2013. False yellowhead (Dittrichia viscosa) causes over infestation with the whitefy pest (Trialeurodes vaporariorum) in tomato crops. International Journal of Agricultural Policy and Research 1(10): 311-318

Parolin, P., Ion Scotta, M, Bresch, C. 2014. Biology of Dittrichia viscosa, a Mediterranean ruderal plant: a review. Phyton (Buenos Aires) 83: 251-262.

Sinden J., Jones, R., Hester, S., Odom, D., Kalisch, C., James, R. 2004. The economic impact of weeds in Australia. Report to the CRC for Australian Weed Management. Technical Series 8: 1-65.

Teillier, S., Macaya, J., Susanna, A., Calleja, J.A. 2014. Volutaria tubuliflora (Murb.) Sennen (Asteraceae), nueva especie alóctona asilvestrada para Chile. Gayana Botánica 71: 276-279

Tixier, M.S., Kreiter, S., Auger, P., Sentenac, G., Salva, G., Weber, M. 2000. Phytoseid mite species located in uncultivated areas surrounding vineyards in three French regions. Acarologia 41: 127-140.

Ugarte, E., Lira, F., Fuentes, N., Klotz, S. 2011. Vascular alien flora, Chile. Check List 7: 365-382.

Zuloaga, F., Morrone, O., Belgrano, M. (Eds.). 2017. Catálogo de las plantas vasculares del cono sur (Argentina, Sur de Brasil, Chile, Paraguay y Uruguay). http://www2.darwin. edu.ar/Proyectos/FloraArgentina/fa.htm. Accedido: Septiembre 2017.

Recibido: 10.03.2017

Aceptado: 25.09.2017 